\title{
The Impact of Pharmacist Care on Diabetes Outcomes in Primary Care Settings: An Umbrella Review of Systematic Reviews
}

\section{Sara Abdulrhim, Sownd Sankaralingam, Mohamed Izham, Ahmed Awaisu* Qatar University, College of Pharmacy, Doha, Qatar *Corresponding author:}

\section{Background}

- Diabetes is a chronic, debilitating illness of an escalating prevalence worldwide ${ }^{1}$

- In Qatar, the prevalence of diabetes is $15.5 \%$ in $2019^{2}$

- By 2030, diabetes will be the seventh cause of death ${ }^{3}$

- Primary care plays a role in diabetes management - a focus in Qatar National Health Strategy 2018-2022

- Several systematic reviews of primary studies evaluating pharmacist's impact on diabetes outcomes in primary care setting were published ${ }^{5,6}$

- Common pitfalls are the focus on limited number of outcomes, inclusion of multiple types of studies, and consideration of a small number of studies

Moreover, clinicians and policymakers have a challenge in decision making using reviews of diverse outcomes

- Therefore, an umbrella review of systematic reviews will compare and contrast the findings of separate reviews

\section{Objectives}

I. To perform a comprehensive review of systematic reviews evaluating pharmacist's impact on diabetesrelated clinical, humanistic, and economic outcomes in primary care settings

II. To synthesize and summarize the main outcomes of pharmacist's interventions in the management of diabetes in primary care settings

\section{Methods}

\section{Data sources}

PubMed, EMBASE, Scopus, Database of Abstracts of Reviews of Effects, Google Scholar, PROSPERO, Cochrane Database of Systematic Reviews, and Joanna Briggs Institute database

Search strategy

Pharmacist + intervention + diabetes + primary care + systematic review

\section{Inclusion criteria}

Reports pharmacist-led or pharmacist-involved intervention Investigates clinical, humanistic, and/or economic outcomes

\section{Screening and selection}

Two reviewers (independently)

\section{Data Extraction}

Data were extracted using a pre-validated data extraction too

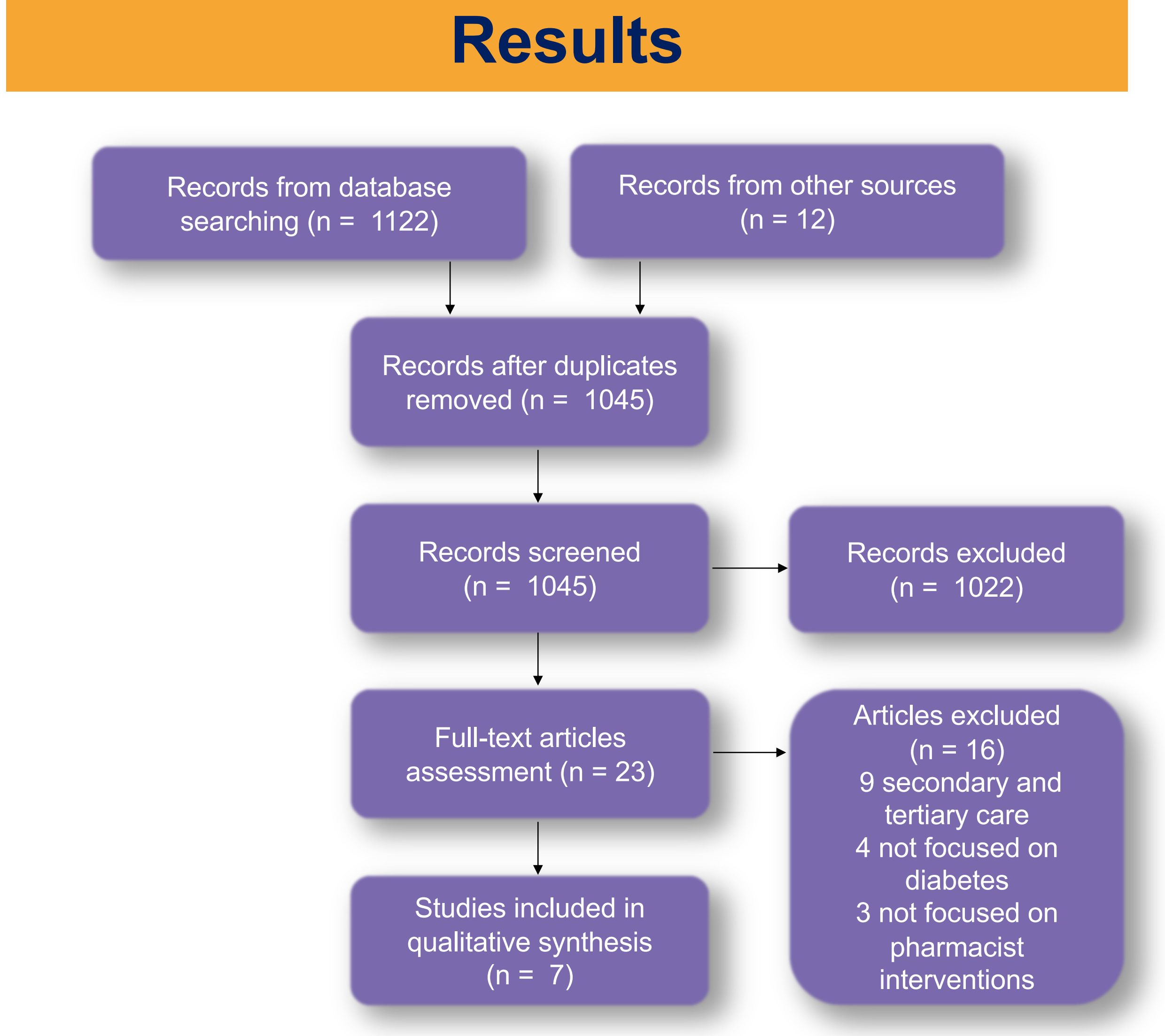

Figure 1. PRISMA flow diagram of the study selection process

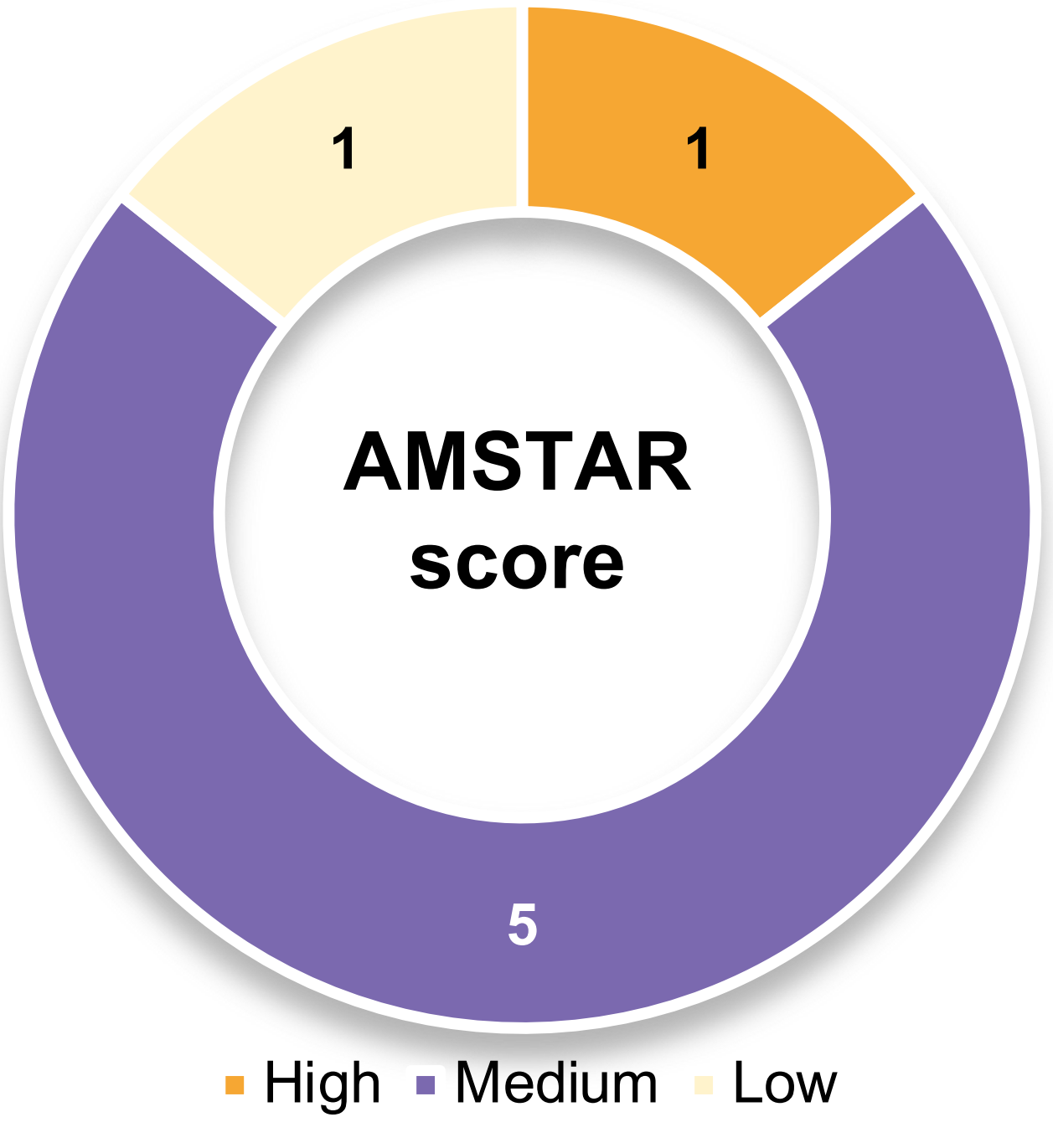

Figure 2. AMSTAR-2 score of the included systematic reviews

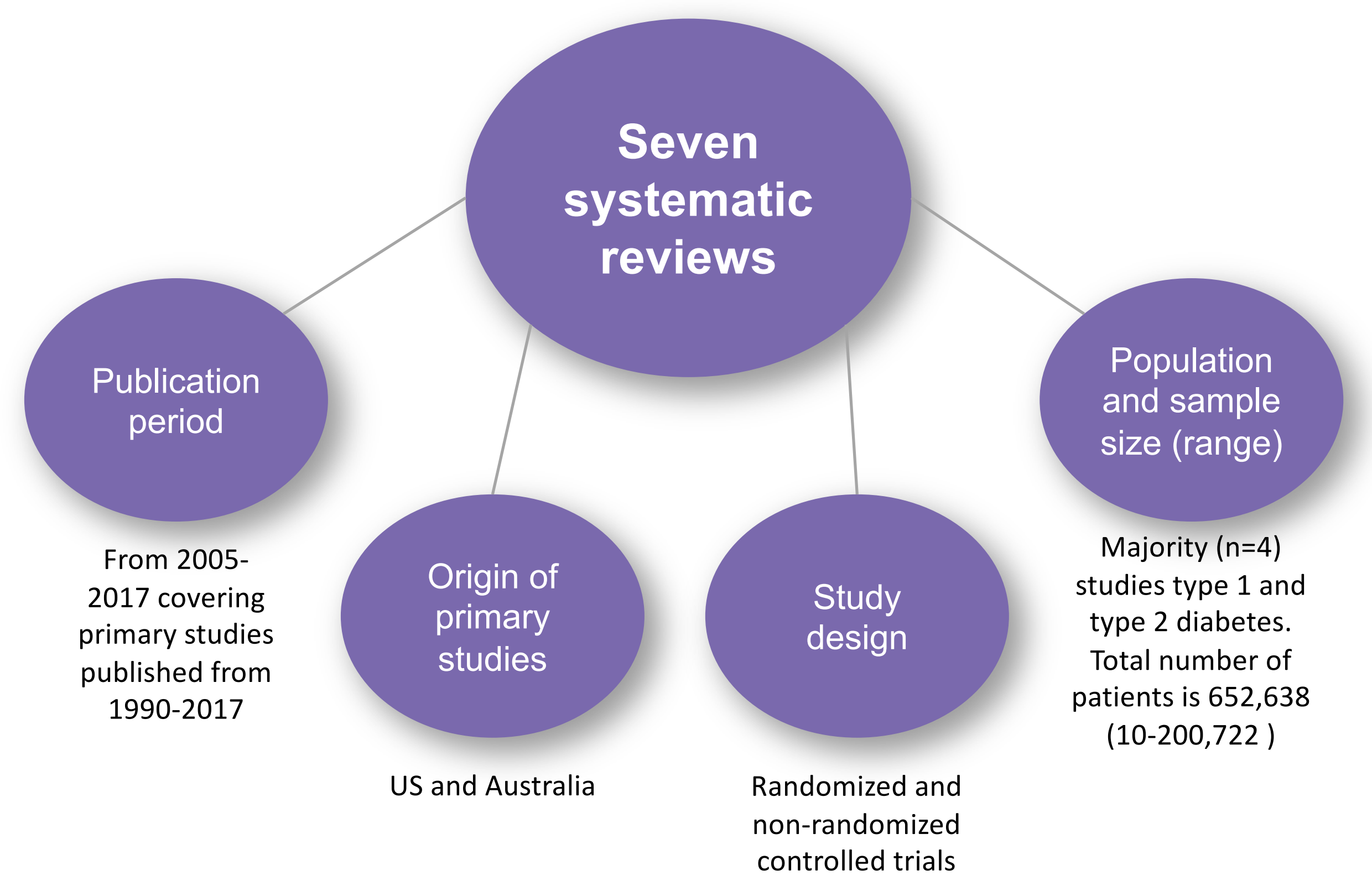

Figure 3. Characteristics of the included systematic reviews

Clinical outcomes

- Improved $\mathrm{HbA}_{1 \mathrm{c}}$ by $0.5-1.1 \%$

- Improved systolic BP by $2.92-7.58 \mathrm{mmHg}$

- Improved diastolic BP by 1.01-6 mmHg

- Improved BMI by $0.19-0.79 \mathrm{~kg} / \mathrm{m}^{2}$

- Improved total cholesterol by $0.24-0.53 \mathrm{mmol} / \mathrm{L}, \mathrm{LDL}$

by $7.1-14.1 \mathrm{mmol} / \mathrm{L}$, HDL by $0.02-0.61 \mathrm{mmol} / \mathrm{L}$

Humanistic outcomes

- No conclusion can be made due to the use of

different assessment tools

Economic outcomes

Benefit-to-cost ratios: 1:1 - 8.5:1

- Benefit-to-cost ratios: 1:1 - 8.5:1

- Cost-saving: $\$ 8$ - $\$ 85,000$ per person per year - Cost-utility: Pharmacist-managed group dominated the usual care group

- Cost of avoiding a diabetes-related cardiovascular

and cerebrovascular event: $\$ 62,803$ and $\$ 114,576$

- Average cost per $1 \% \mathrm{HbA}_{1 \mathrm{c}}$ reduction: $\$ 174 /$ person

Figure 4. Impact of pharmacist's interventions on ECHO model
Results (Cont'd)

Below is a summary of the reported impact of pharmacist's interventions by the 7 systematic reviews

1. Providing patient and family education about diabetes self-care, lifestyle changes, exercise, smoking cessation, medication adherence, and medication side effects

2. Performing drug therapy management

3. Clinically assessing patients, reviewing medications, and reviewing medical health records

4. Training patients on the use of blood glucose meters

5. Following patients up regularly

6. Prescribing medications

7. Preventing diseases through immunization provision

8. Evaluating drug therapy

9. Communicating with other healthcare professionals and aiding clinical decision-making through providing therapeutic recommendations

\section{Limitations}

- There is a high heterogeneity among the primary studies as a result of the variations in original studies setting, number of patients, type of pharmacist's interventions, and outcomes measures

Each systematic review stratified the primary studies differently (based on study setting, disease, outcome, or not stratified)

\section{Conclusion}

- Various pharmacist's interventions provided to patients with type 1 or type 2 diabetes in primary care setting were shown to improve clinical, humanistic, and economic outcomes.

- There is a need to develop a comprehensive conceptual framework regarding the types of pharmacist's interventions that target patients with diabetes attending primary care facilities, their families, and healthcare systems

\section{References}

Zimmet, P., Alberti, J. Global and societal implications of the diabetes epidemic

. 2019. World Health Organization

Fazel, M., Bagalagel, Ategy 2018-2022. Moph.gov.qa. 2018. Diabetes Care by Pharmacists as Part of Health Care Team in Ambulatory ns: A Systematic Review and Meta-analysis. Annals of Pharmacotherapy

Blenkinsopp, A. and Hassey, A. (2005). Effectiveness and acceptability of community pharmacy-based interventions in type 2 diabetes: a critical review of intervention design, pharmacist and patient perspectives. International Journ 